\title{
Megaesôfago em cães
}

\author{
Canine Megaesophagus
}

\section{Neide Mariko Tanaka ${ }^{[a]}$, Nayara Hoogevonink ${ }^{[\mathrm{b}]}$, Ângela Patrícia Tucholski ${ }^{[\mathrm{b}]}$, Silvia Manduca Trapp ${ }^{[\mathrm{c}]}$, Michele Salmon Frehse ${ }^{[\mathrm{d}]}$}

[a] Médica veterinária, docente Ph.D da Universidade do Norte do Paraná (UNOPAR), Arapongas, PR e Universidade Tuiuti do Paraná (UTP), Curitiba, PR- Brasil, e-mail: nmtanaka@onda.com.br

[b] Médica veterinária, Curitiba, PR-Brasil e-mail: nayarahk@hotmail.com; angela_tuc@hotmail.com

[c] Médica veterinária, docente Doutor da Universidade do Norte do Paraná (UNOPAR), Arapongas, PR - Brasil, e-mail: smanducatrapp@gmail.com

[d] Médica veterinária, Doutoranda, Universidade Estadual de Londrina (UEL), Londrina, PR - Brasil, e-mail: mi_uel2003@yahoo.com.br

\section{Resumo}

Megaesôfago é o termo que se refere à dilatação e à hipomotilidade esofágica, resultante de distúrbio primário ou secundário. É classificado em congênito, adquirido idiopático e adquirido secundário. Constitui a principal causa de regurgitação através da boca ou das narinas, pois a hipomotilidade resulta em acúmulo e retenção de alimento e líquido no esôfago. Outras complicações associadas são tosse, corrimento nasal mucupurulento, dispneia, pneumonia por aspiração e emagrecimento progressivo. Tem sido descrito em algumas raças de cães, como no Fox Terrier, Schnauzer, Pastor Alemão, Dog Alemão, Golden Retriever e Setter Irlandês, e em gatos é relativamente rara, sendo a raça Siamesa a mais predisposta. O diagnóstico baseia-se na análise dos sinais clínicos, anamnese e exames complementares como radiografias, esofagograma contrastado, endoscopia e cintilografia. O tratamento depende da etiologia, porém baseia-se em cuidados no fornecimento do alimento. A terapia medicamentosa está associada com as complicações. Até o momento, não há cura ou tratamento clínico que solucione a debilidade esofágica congênita; já no megaesôfago adquirido secundário há resolução, ou pelo menos melhora, quando a causa subjacente for tratada com sucesso. O prognóstico é ruim. A mortalidade está relacionada à pneumonia por aspiração, à falta de concordância com o proprietário e à desnutrição. Os procedimentos cirúrgicos não fazem parte da prática clínica. A técnica da plicatura na parede esofágica foi criada por Meyer (1910) para redução do diâmetro luminal, através do pregueamento do tecido dilatado. O objetivo desta revisão é discutir o diagnóstico e os procedimentos clínicos e cirúrgicos, visando à orientação e a conduta diante do megaesôfago canino.

Palavras-chave: Megaesôfago. Diagnóstico. Clínico. Cirúrgico. Tratamento. 


\begin{abstract}
Megaesophagus is the term that refers to esophageal dilation and hypomotility resulting from primary or secondary disorder. It is classified as congenital, acquired idiopathic and secondary acquired. It is the main cause of regurgitation through the mouth or nostrils, for hypomotility resulting in accumulation and retention of food and fluid in the esophagus. Other complications are associated with cough, runny nose mucupurulent, dyspnea, aspiration pneumonia and progressive weight loss. It has been described in some breeds, like the Fox Terrier, Schnauzer, German Shepherd Dog German, Golden Retriever and Irish Setter in cats are relatively rare, the Siamese bring more predisposed. The diagnosis is based on the analysis of clinical signs, history and tests such as radiographs, contrast esophagogram, endoscopy and scintilography. Treatment depends on the etiology but based on care in providing the food. Drug therapy is associated with complications. So far, there is no cure or medical treatment that addresses the weakness of esophageal disease; for the acquired secondary megaesophagus there is solution, or at least an improvement, when the underlying cause is successfully treated. The prognosis is poor. Mortality is related to aspiration pneumonia, lack of agreement with the owner and malnutrition. Surgical procedures are not part of clinical practice. The technique of lacation in the esophageal wall was created by Meyer (1910) to reduce the luminal diameter, by folding the tissue stretched. The purpose of this review is to discuss the diagnosis and clinical and surgical procedures aimed at the orientation and postures toward canine megaesophagus.
\end{abstract}

Keywords: Megaesophagus. Diagnostic. Clinical. Surgical. Treatment.

\title{
Introdução
}

O megaesôfago caracteriza-se pela ausência ou diminuição acentuada dos plexos nervosos intramurais do esôfago, determinando distúrbio motor esofágico à deglutição. Quando esta destruição celular atinge níveis de 50\% a 95\%, ocorre uma progressiva desorganização de toda a atividade motora e a dilatação do órgão (CELANO et al., 2007). A motilidade esofágica fica reduzida ou ausente, resultando no acúmulo ou na retenção de alimento e de líquido no esôfago (LONGSHORE, 2008). Essa dilatação do esôfago resulta em uma severa desordem na motilidade, tornando o órgão dilatado e flácido e o peristaltismo ineficiente (WASHABAU; HOLT, 2003). O objetivo desta revisão é discutir o diagnóstico e os procedimentos clínicos e cirúrgicos visando à orientação e à conduta ante o megaesôfago canino.

\section{Desenvolvimento}

\section{Esôfago e megaesôfago}

A principal função do esôfago é o transporte de líquidos e sólidos ingeridos da cavidade oral até o estômago. As estruturas anatômicas que permitem esta função são representadas pelos músculos estriados do esfíncter superior do esôfago, pelos músculos estriados e lisos do corpo esofágico e pelo músculo liso do esfíncter do esôfago (WASHABAU, 2004). Sua ação é controlada por mecanismos reflexos, principalmente pelo nervo vago. Conforme o alimento passa pela faringe, estimula os mecanismos de peristaltismo no esôfago, contraindo as porções superiores e relaxando as porções inferiores, fazendo com que o fluxo seja unidirecional em direção ao estômago (BONFADA, 2005). As porções torácicas, cervical e proximal do esôfago situam-se à esquerda da linha média, porém desde a bifurcação traqueal até o estômago localiza-se ligeiramente à direita (HEDLUND, 2002).

A parede do esôfago é composta por quatro camadas: adventícia, muscular (com dois níveis oblíquos), submucosa e mucosa. Não há camada serosa como em outras partes do trato gastrintestinal, fazendo com que se torne o local mais propício para deiscência de sutura, pois a selagem com fibrina é mais lenta após uma lesão (SHELTON, 1998; HEDLUND, 2002; OLSEN, 2002; SHELTON, 2003; BONFADA, 2005). Mas, 
segundo ressaltou Shelton $(1998,2003)$, a porção torácica esofágica é revestida em ambos os lados pela pleura mediastinal, que pode funcionar como fonte de células mesoteliais e fibrina na vedação das feridas esofágicas.

A vascularização da porção cervical do esôfago é derivada da artéria tireóidea cranial e caudal. Aproximadamente dois terços proximais do esôfago torácico são supridos pelos ramos broncoesofágicos da aorta, porém o terço final é suprido pelos ramos aórtico, intercostais e pelos ramos esofágicos distais da artéria gástrica esofágica esquerda (HEDLUND, 2002), além de uma rede vascular intramural na camada submucosa, que, segundo Shelton $(1998,2003)$ e Hedlund (2002), irão garantir a vascularização adequada para cicatrização cirúrgica.

O reflexo de motilidade esofágica começa quando o alimento estimula neurônios sensoriais aferentes na mucosa esofágica, a qual envia mensagens para o centro da deglutição no tronco cerebral, via nervo vago. Mensagens eferentes dos motoneurônios inferiores no núcleo ambíguo são enviadas pelo nervo vago e estimulam a contração dos músculos liso e estriado do esôfago. Lesões em qualquer parte deste caminho, incluindo a junção mioneural, podem resultar em hipomotilidade e distensão esofágica (SPILLMANN, 2007; TILLEY; SMITH, 2008).

O megaesôfago caracteriza-se pela ausência ou pela diminuição acentuada dos plexos nervosos intramurais do esôfago, determinando distúrbio motor esofágico à deglutição. Quando esta destruição celular atinge níveis de $50 \%$ a $95 \%$, ocorre uma progressiva desorganização de toda a atividade motora e dilatação do órgão (CELANO et al., 2007). A motilidade esofágica fica reduzida ou ausente, resultando no acúmulo ou na retenção de alimento e de líquido no esôfago (LONGSHORE, 2008). Esta dilatação do esôfago resulta em uma severa desordem na motilidade, tornando o órgão dilatado e flácido e o peristaltismo ineficiente (WASHABAU; HOLT, 2003).

A afecção pode ser congênita, idiopática ou secundária adquirida, e também têm sido descritas predisposições hereditárias em algumas raças de cães, como no Fox Terrier, Schnauzer, Pastor Alemão, Dog Alemão, Golden Retriever e Setter Irlandês. Em gatos é relativamente rara, talvez graças à prevalência de fibras musculares lisas no esôfago felino, sendo a raça Siamesa a mais predisposta (ANDRADE, 2007).

$\mathrm{O}$ megaesôfago congênito corresponde à hipomotilidade e à dilatação generalizada do esôfago, provocando regurgitação e subdesenvolvimento do filhote após o desmame. A patogenia da forma congênita ainda não está completamente esclarecida, embora estudos apontem para um defeito na inervação aferente vagal para o estômago (WASHABAU, 2004). Mas segundo Willard (2006), a causa ainda é desconhecida e não há evidências de desmielinização ou degeneração neural e a inervação vagal eferente parece estar normal (WILLARD, 2006).

O megaesôfago secundário adquirido ocorre como consequência de causas primárias que provocam alterações motoras no esôfago ou no esfíncter gastroesofágico, determinando sua dilatação passiva. As principais causas de megaesôfago secundário são miastenia grave, lúpus eritematoso, polimiosite, polineurite, neuropatias degenerativas, hipoadrenocorticismo, hipotireoidismo, déficit de tiamina, intoxicações por metais pesados (chumbo e tálio), tumores (principalmente timoma) e problemas cervicais (ANDRADE, 2007). De acordo com Gaiga, Pigatto e Brun (2006), um caso de megaesôfago causado por tétano em um cão foi relatado. No magaesôfago adquirido secundário pode-se observar fraqueza, paresia ou paralisia, ataxia, náusea, disfagia, dor ou depressão.

De acordo com Washabau (2004), a miastenia grave do animal é responsável por pelo menos 25\% dos casos secundários. Em alguns casos da miastenia grave, a regurgitação e a perda de peso podem ser apenas os sinais de manifestação da enfermidade, enquanto na maioria das ocorrências de megaesôfago secundário adquirido, a regurgitação é meramente um dos vários sinais clínicos.

Não há etiologia conhecida para a maioria dos casos de megaesôfago que se iniciam na fase adulta, os quais são denominados megaesôfago adquirido idiopático. A síndrome é de ocorrência espontânea em cães adultos com 7 a 15 anos de idade, sem predileção por raça ou sexo. E essa forma é a principal causa de regurgitação em cães. Esse distúrbio é comparado, erroneamente, com a acalasia esofágica em humanos. A acalasia é uma deficiência do relaxamento do esfíncter esofágico inferior e de peristalse ineficiente do corpo esofágico. Um distúrbio semelhante nunca foi comprovado em cães. Observaram-se várias diferenças importantes entre megaesôfago idiopático no cão e acalasia em seres humanos. As pesquisas mais recentes

Rev. Acad., Ciênc. Agrár. Ambient., Curitiba, v. 8, n. 3, p. 271-279, jul./set. 2010 
sugerem um defeito na resposta neural aferente à distensão do esôfago. As respostas dos esfíncteres esofágicos superiores e inferior para a deglutição parecem permanecer intactas, mas a distensão do esôfago não inicia as contrações peristálticas nos animais acometidos. Ainda não se determinou o local exato desta anormalidade na resposta neural aferente (WASHABAU; HOLT 2003; WASHABAU, 2004).

\section{Sinais clínicos}

Os sinais clínicos da doença são caracterizados pela regurgitação de alimento e água, perda de peso ou crescimento deficiente, hipersalivação e som de borbulhas a deglutição. Outras alterações também podem ser observadas no megaesôfago, como tosse, corrimento nasal mucopurulento e dispneia com pneumonia por aspiração concomitante. Os achados no exame físico são: regurgitação, perda de peso, auscultação de líquidos e alimentos retidos no esôfago, halitose, ptialismo, saliência do esôfago na entrada torácica e dor associada à palpação da região do esôfago. Relacionadas às causas ou às sequelas do megaesôfago são crepitações respiratórias, taquipneia, pirexia, mialgia, fraqueza muscular, atrofia muscular, hiporreflexia, déficits proprioceptivos e posturais, distúrbios anatômicos (midriase com perda de reflexo pupilar à luz, mucosas nasal e ocular ressecadas), déficits de nervos cranianos (especialmente os nervos VI, IX e X), paresia ou paralisia e alterações da consciência (LONGSHORE, 2008).

De acordo com German (2005), a principal manifestação clínica de megaesôfago é regurgitação. Mas essa regurgitação deve ser diferenciada de vômito, de náusea e de disfagia. A regurgitação difere do vômito, caracterizando-se por uma eliminação retrógrada passiva do alimento não digerido a partir do esôfago. $\mathrm{O}$ vômito caracteriza-se pelas atividades coordenadas dos sistemas gastrintestinais, musculoesqueléticos e nervoso, culminando na eliminação ativa do alimento digerido ou parcialmente digerido pelo trato gastrintestinal.

Segundo Washabau (2004), a frequência da regurgitação pode variar de um episódio a cada poucos dias a vários episódios por dia. A regurgitação associada ao megaesôfago ocorre de vários minutos a horas após a alimentação, enquanto a regurgitação associada aos distúrbios orofaríngeos ou cricofaríngeos geralmente se manifesta imediatamente após a alimentação.

\section{Diagnóstico}

De acordo com Spillmann (2007), o diagnóstico começa com um minucioso exame físico da cavidade oral, região cervical e ausculta do tórax, a fim de se pesquisar transtornos orofaríngeos, dilatação ou "massa" na região cervical na porção do esôfago e sinais de pneumonia por aspiração como uma complicação das doenças esofágicas.

Os exames hematológicos, o bioquímico sérico e a urinálise devem ser realizados em todos os casos para se pesquisar possíveis causas secundárias de megaesôfago (WASHABAU, 2004). Como a hiponatremia e a hipercalemia, que podem sugerir hiperadrenocorticismo, a hipercolesterolemia pode estar geralmente associada ao hipotireoidismo e a creatina cinase elevada pode sugerir distúrbio muscular primário (LONGSHORE, 2008).

$\mathrm{Na}$ ausência de doenças metabólicas ou sistêmicas, a hipoproteinemia (associada à má nutrição) e a leucocitose (associada à inflamação esofágica ou à pneumonia por aspiração) são as únicas alterações dos exames laboratoriais que são ocasionalmente encontrados (WASHABAU, 2004).

Outros testes laboratoriais que podem ser realizados são:

a) títulos de anticorpos receptores da acetilcolina, para fazer a triagem da adquirida miastenia grave, devendo ser realizados em todos os pacientes com megaesôfago;

b) títulos do anticorpo antinuclear, para avaliar lúpus eritematoso sistêmico (LER);

c) estimulação do hormônio adrenocorticotrópico (ACTH), para avaliar a função adrenal;

d) nível de tiroxina $\left(\mathrm{T}_{4}\right)$ - hormônio estimulante da tireoide ( $\left.\mathrm{TSH}\right)$ - livre, para avaliar a função tireóidea;

e) níveis sanguíneos de chumbo e de colinesterase para avaliar intoxicação (LONGSHORE, 2008).

Rev. Acad., Ciênc. Agrár. Ambient., Curitiba, v. 8, n. 3, p. 271-279, jul./set. 2010 
Radiografia simples, radiografia contrastada e endoscopia são os métodos diagnósticos atualmente disponíveis na clínica veterinária. A radiografia simples da região cervical e do tórax deve ser realizada em todos os animais com suspeita de doença esofágica. As radiografias torácicas indicarão alguma das complicações da doença esofágica, incluindo a pneumonia por aspiração, a efusão pleural, a mediastinite e o pneumotórax (WASHABAU, 2004). Nas radiografias simples do pescoço, o esôfago aparece dilatado com acúmulo de gás, fluido ou ingesta, e a traquéia estará quase sempre deslocada ventralmente pelo esôfago distendido (LANGSHORE, 2008).

Segundo Spillmann (2007), a radiografia contrastada é indicada quando a radiografia simples não consegue fechar o diagnóstico. O sulfato de bário ou o contraste contendo iodo são utilizados. Este último é usado especialmente quando há suspeita de perfuração esofágica ou fístula traqueoesofágica. Quando se tem dilatação intramural ou estenose de extensão, pode-se visualizá-la por meio da mistura entre o alimento e o contraste. Durante este procedimento deve-se ter cuidado para que o paciente não aspire o conteúdo, quando posicionado para a radiografia.

A endoscopia do esôfago tornou-se um método muito útil no diagnóstico e no tratamento da doença esofágica. É frequentemente realizada após a avaliação radiográfica simples e em vários casos pode ser substituído pela radiografia contrastada. Em contrapartida, Willard (2006) descreve que a endoscopia não é tão útil quanto as radiografias contrastadas para o diagnóstico.

Segundo Simpson (2008), os achados anormais na endoscopia incluem: presença de alimentos, fluido, parasita, corpos estranhos, perfurações, constrições secundárias ao anel vascular, estenoses esofágicas, massas, aversão da mucosa gástrica no esôfago (intussuscepção gastroesofágica) e redundância da mucosa do esôfago, que pode sugerir dilatação do esôfago, apesar de que muitas vezes é difícil sua confirmação por meio da endoscopia.

Outra limitação é que a anestesia pode provocar um relaxamento da musculatura estriada, no esôfago e, por conseguinte, ser mal interpretada como dilatação (SPILLMANN, 2007).

\section{Tratamento}

Até o momento, não há cura ou tratamento clínico que solucione a debilidade esofágica congênita. Indica-se um tratamento dietético conservador, a fim de evitar o agravamento da dilatação e a aspiração. Classicamente, o animal é alimentado com alimentação pastosa, em uma plataforma elevada que requeira o animal em estação, com o apoio dos membros posteriores. Desta maneira, o esôfago cervical e torácico permanece em posição vertical quando o alimento é ingerido, o que permite que a gravidade auxilie a passagem do alimento através do esôfago para o estômago. Esta posição deve ser mantida por cinco a dez minutos após a alimentação. Oferecer várias refeições por dia em pequenas quantidades também evita a retenção de alimento no esôfago (WILLARD, 2006).

Alguns animais apresentam melhor resposta recebendo alimentação seca ad libitum durante o dia, numa plataforma elevada. Não se pode prever se um determinado animal obterá mais beneficio recebendo alimento seco ou líquido, sendo necessárias, portanto, várias tentativas e erros para se determinar a melhor dieta para um animal em particular. Em alguns cães, o esôfago dilatado pode retornar parcialmente ao tamanho e função normais. Ainda que o esôfago permaneça dilatado, alguns animais podem ter boa qualidade de vida, com o adequado manejo nutricional (WILLARD, 2006).

Os animais que não podem manter equilíbrio nutricional adequado com a ingestão oral devem ser alimentados mediante uso de sonda de gastrostomia temporária ou permanente. Esses tubos podem ser colocados mediante cirurgia ou por via percutânea, com o auxilio de um endoscópio (WASHABAU, 2004). Segundo German (2005), em curto ou em médio prazo, a alimentação assistida pode ser benéfica aos pacientes com megaesôfago, uma vez que permite melhorias na condição corporal do paciente e dá tempo para se adaptarem às alterações na alimentação oral.

No megaesôfago adquirido secundário, a resolução, ou pelo menos a melhora, poderá ocorrer se a causa subjacente for tratada com sucesso. Por exemplo, se o hipoadrenocorticismo estiver presente, o 
tratamento envolve a administração de esteroide como glicocorticoides e mineralocorticoides. Nos casos em que são secundárias à miastenia grave, devem ser tratados com a combinação de um anticolinesterásicos $\left(\right.$ piridostigmina $^{\circledR}$ ) e de glicocorticoides (prednisona ${ }^{\circledR} /$ prednisolona $^{\circledR}$ ), cuja utilização é controversa, pois pode agravar a fraqueza muscular e aumentar o risco de aspiração e pneumonia (GERMAN, 2005).

De acordo com Washabau (2004), deve-se identificar as infecções pulmonares mediante cultivo e teste de sensibilidade antimicrobiana, selecionando-se um antibiótico adequado para o(s) microrganismo(s) causador(es). A amostra para exame pode ser obtida do lavado traqueal ou broncoalveolar no momento da endoscopia.

Os tratamentos clínicos devem estimular o peristaltismo esofágico, como a metoclopramida ou cisaprida, ou diminuir o tônus do esfíncter esofágico inferior, como os anticolinérgicos ou antagonistas de canais de cálcio, nos animais acometidos. A metoclopramida e a cisaprida são agentes procinéticos de músculos lisos que provavelmente não atuam nos músculos estriados do esôfago dos cães, pois não apresentam receptores. Em virtude dos seus conhecidos efeitos procinéticos no tônus do esfíncter esofágico inferior, a cisaprida pode reduzir a taxa de trânsito esofágico no cão. Desse modo, não se deve recomendar a cisaprida no tratamento do megaesôfago idiopático de cães, a menos que haja distúrbios do esvaziamento gástrico concomitante. Os antagonistas de canais de cálcio apresentam efeitos hipotensivos potentes na musculatura lisa vascular, porém pouco efeito no músculo liso do esfíncter do esôfago dos cães. A administração de anticolinérgicos está associada a vários efeitos colaterais, prejudicando a sua utilidade clínica (WASHABAU, 2004).

Nenhuma medicação é atualmente utilizada como medicamento único para tratar o megaesôfago, mas pode dirigir o tratamento para as causas subjacentes ou condições associadas como, por exemplo, o sucralfato $\left(0,5-1,0 \mathrm{~g} /\right.$ cão per os a cada $8 \mathrm{~h}$ ), bloqueadores de $\mathrm{H}_{2}$ (por exemplo, famitidina, $0,5 \mathrm{mg} / \mathrm{kg}$ per os a cada $12-24 \mathrm{~h}$ nos cães) ou omeprazol $(0,7 \mathrm{mg} / \mathrm{kg}$ per os a cada $24 \mathrm{~h}$ nos cães) podem ser utilizados se houver esofagite de refluxo. A metoclopramida $(0,2-0,5 \mathrm{mg} / \mathrm{kg}$ per os a cada $6-8 \mathrm{~h}$ nos cães) acelera o esvaziamento gástrico, aumentando o tônus do esfíncter gastroesofágico e é mais valiosa quando a esofagite por refluxo for contribuinte ou a causa primária, mas a sua utilização para outras causas pode ter sucesso limitado. Antibióticos de amplo espectro são necessários para pacientes com pneumonia por aspiração, e para pacientes com regurgitação podem ser usadas a via parenteral ou administração entérica via sonda de gastrotomia (LONGSHORE, 2008).

\section{Cirurgias para correção do megaesôfago}

Suspeitas de que o megaesôfago poderia ser uma acalasia similar à do homem, fez com que fosse tratado cirurgicamente com a técnica de esofagomiotomia de Heller modificada. Mesmo que essa técnica reduza efetivamente a pressão no esfíncter, favorecendo o esvaziamento esofágico, ela representa um alto risco operatório, com 38\% de mortalidade nos pós-operatórios e baixos resultados. As complicações operatórias incluem refluxo gastresofágico e esofagites. A cardioplastia de Wendel apresenta o mesmo problema de refluxo gástrico (TORRES, 2000).

Torres (2000) descreveu a técnica cirúrgica denominada cardioplasia esofágica diafragmática em dez cães portadores de megaesôfago congênito, cujo mecanismo de ação se deve à atividade contrátil do diafragma durante os ciclos respiratórios, fazendo com que pequenas quantidades de alimento entrem no estômago. Segundo o mesmo autor, esta técnica obtém resultados positivos, como a recuperação do estado nutricional e melhora no transporte esofagiano dos animais acometidos.

Para Washabau (2004), a técnica de esofagomiotomia de Heller modificado pode não ser recomendada no tratamento do distúrbio, pois o esfíncter inferior do esôfago é normotensivo e relaxa adequadamente durante a deglutição nos animais acometidos.

Cirurgicamente, nenhum método melhora a motilidade esofágica. No megaesôfago adquirido secundário faz-se a cirurgia de correção das anomalias de anel vascular; já a esofagotomia e a esofagoectomia são utilizadas para remover corpos estranhos ou neoplasia esofágica. Reisinger (1907 apud WASHABAU, 2004) criou uma técnica para reduzir o diâmetro do esôfago na acalásia, retirando uma faixa da parede do esôfago, por esofagoectomia.

Rev. Acad., Ciênc. Agrár. Ambient., Curitiba, v. 8, n. 3, p. 271-279, jul./set. 2010 
A cardiomiotomia de Heller reduz o tônus esofágico inferior e pode melhorar o movimento facilitado pela gravidade da ingesta para dentro do estômago (FINGEROTH, 1998; TORRES, 2000; HEDLUND, 2002; TILLEY; SMITH, 2008).

A plicatura na parede esofágica foi criada por Meyer (1910 apud WASHABAU, 2004), para redução do diâmetro luminal, por meio do pregueamento do tecido dilatado, com suturas franzidas do tipo de Lembert, sem invadir o lúmen. Recentemente o procedimento voltou a ser utilizado nos casos de megaesôfago avançado como complemento da cardiomiotomia. Fingeroth (1998) ressaltou que embora o pregueamento possa fazer com que o esôfago pareça melhor, este não altera a capacidade de fazer a propulsão aboral do material ingerido, aumentando o risco de complicações, ou seja, é improvável que gere qualquer benefício clínico para o paciente. Fingeroth (1998) também contesta a técnica em animais, pois, segundo estudos, gera uma regurgitação intratável, além do risco de contaminação.

Em experimentos com suínos, Azevedo et al. (2004) testaram o uso da plicatura gástrica na miototomia esofágica por laparoscopia, visando ao tratamento de megaesôfago refratário, observando atraso na cicatrização, intervenção mais demorada, descontinuidade na musculatura esofagiana incisada, bem como o aumento luminal, não obtendo resultados satisfatórios .

Em seres humanos, Valezi et al. (2004) relataram a eficácia da cardiomiotomia com fundoplicatura parcial por vídeo-laparoscopia (Heller-Dor) no tratamento de 12 pacientes portadores de megaesôfago grau II. Herbella et al. (1999), em estudo retrospectivo de 83 casos, de 1977 a 1995, também atestam que a cardiomiotomia associada à fundoplicatura é a cirurgia de escolha para o tratamento do megaesôfago, mostrando ser uma operação segura e com bons resultados.

Medeiros (2005) relatou métodos de tratamento cirúrgico para o megaesôfago em seres humanos, desde a simples supressão da dificuldade do esvaziamento esofágico até a criação de um mecanismo valvular que impeça o refluxo gastroesofágico, fornecendo opções de tratamento adequado ao seu grau de evolução.

\section{Prognóstico}

O prognóstico para megaesôfago idiopático congênito em animais é razoável. Com a atenção adequada às necessidades calóricas e aos episódios de pneumonia por aspiração, vários animais apresentam melhora da motilidade esofágica ao longo dos meses (GERMAN, 2005).

A morbidade e a mortalidade decorrentes do megaesôfago adquirido permanecem inaceitavelmente elevadas. Vários animais eventualmente sucumbem aos efeitos da subnutrição crônica e ao episódio repetido de pneumonia por aspiração. Deve-se atribuir prognóstico desfavorável em tais casos (SPILLMANN, 2007).

Os animais com megaesôfago adquirido secundário apresentam prognósticos mais favoráveis caso a doença primária possa ser prontamente identificada e tratada com êxito. Os casos refratários resultam de distensão esofágica crônica, degeneração de nervos mioentéricos e atrofia muscular (WASHABAU, 2004). Segundo Longshore (2008), a pneumonia e a má nutrição são as causas que podem levar à morte.

\section{Considerações finais}

O megaesôfago é resultante de alteração no peristaltismo esofágico, produzindo uma alteração na motilidade, que fica diminuída ou ausente, resultando essa alteração no acúmulo de alimento e de líquido no esôfago e, consequentemente, distendendo o órgão.

A forma idiopática do megaesôfago é a causa mais comum, podendo ser congênita, sendo mais comum em filhotes, e a adquirida mais comum em animais idosos. A forma adquirida ocorre secundariamente a várias doenças sistêmicas. Até o momento, não há cura ou tratamento clínico que solucione a debilidade esofágica congênita, mas se indica um tratamento dietético conservador, a fim de se evitar o agravamento da dilatação e a aspiração.

Rev. Acad., Ciênc. Agrár. Ambient., Curitiba, v. 8, n. 3, p. 271-279, jul./set. 2010 
Procedimentos cirúrgicos como a cardiomiotomia de Heller reduzem o tônus esofágico inferior e podem melhorar o movimento facilitado pela gravidade da ingesta para dentro do estômago, porém, nenhum método melhora a motilidade esofágica. Embora o pregueamento seja indicado nos casos de megaesôfago avançado e possa fazer com que o esôfago pareça melhor, não altera a capacidade de fazer a propulsão aboral do material ingerido, aumentando o risco de complicações, ou seja, é improvável que gere qualquer benefício clínico para o paciente.

\section{Referências}

ANDRADE, S. F. Megaesôfago secundário à miastenia grave em uma cadela da raça Pastor Alemão. Semina: Ciências Agrárias, v. 28, n. 3, p. 477-482, 2007.

AZEVEDO, J. L. M. C. et al. Cicatrização da miotomia esofagiana por acesso videolaparoscópico com e sem plicatura gástrica associada em porcos. Revista Brasileira de Videocirurgia, v. 2, n. 4, p. 177-183, 2004.

BONFADA, A. T. Cirurgia torácica vídeo assistida sem intubação seletiva com acesso modificado para sutura do esôfago caudal em cães. 2005. 72 f. Dissertação (Mestrado em Cirurgia Veterinária) - Setor de Ciências Agrárias, Universidade Federal de Santa Maria, Santa Maria, 2005.

CELANO, R. M. G. et al. Avaliação nutricional pré-operatória dos pacientes com megaesôfago não-avançado. Revista do Colégio Brasileiro de Cirurgiões, v. 34, n. 3, p. 25-31, 2007.

FINGEROTH, J. M. Técnicas cirúrgicas para as afecções do esôfago. In: SLATTER, D. Manual de cirurgia de pequenos animais. 2. ed. São Paulo: Manole, 1998. p. 667-681.

GAIGA, L. H.; PIGATTO, J. A. T.; BRUN, M. V. Megaesôfago e hérnia de hiato esofágico associados ao tétano em um cão: relato de caso. Revista da Faculdade de Zootecnia Veterinária e Agronomia, v. 13, n. 2, p. 145-152, 2006.

GERMAN, A. J. How treat megaesophagus. In: NORTH AMERICAN VETERINARY CONFERENCE, $19 ., 2005$. Orlando. Proceedings... Orlando: North American Veterinary Conference, 2005.

HEDLUND, C. S. Cirurgia do aparelho digestório. In: FOSSUM, T. W. Cirurgia de pequenos animais. São Paulo: Roca, 2002. p. 259-289.

HERBELLA, F. A. M. et al. Resultados tardios da operação de Heller associada à fundoplicatura no tratamento do megaesôfago: análise de 83 casos. Revista da Associação Médica Brasileira, v. 45, n. 4, p. 317-322, 1999.

LONGSHORE, R. C. Megaesôfago. In: TILLEY, L. P.; SMITH, F. W. K. Consulta Veterinária em 5 minutos: canina e felina. 3. ed. São Paulo: Manole, 2008. p. 950-951.

MEDEIROS, S. C. Tratamento cirúrgico do megaesôfago. 2005. 54 f. Monografia (Trabalho de conclusão de residência médica em cirurgia geral) - Hospital Geral de Jacarepaguá, RJ, Rio de Janeiro, 2005.

OLSEN, D. Non-cardiac thoracic surgery. In: WESTERN VETERINARY CONFERENCE, 74., 2002, Las Vegas. Proceedings... Las Vegas: Annual Western Veterinary Conference, 2002. Disponível em: <http://www.vin.com/ Members/Proceedings/Proceedings.plx?CID= wvc2002\&PID=pr00955\&O=VIN>. Acesso em: 20 nov. 2009.

SHELTON, G. D. Distúrbios neuromusculares da deglutição. In: SLATTER, D. Manual de cirurgia de pequenos animais. 2. ed. São Paulo: Manole, 1998. p. 646-650.

SHELTON, G. D. Distúrbios musculares e de junção neuromuscular. In: BIRCHARD, S. J.; SHERDING R. G. Manual Saunders de clínica de pequenos animais. 2. ed. São Paulo: Roca, 2003. p. 1438.

SIMPSON, K. W. Diagnostic gastrointestinal endoscopy. In: WORLD SMALL ANIMAL VETERINARY CONGRESS, 33., 2008, Dublin. Proceedings... Dublin: World Small Animal Veterinary Congress, 2008.

Rev. Acad., Ciênc. Agrár. Ambient., Curitiba, v. 8, n. 3, p. 271-279, jul./set. 2010 
SPILLMANN, T. Esophageal diseases diagnostic and therapeutic approach. In: ANNUAL WSAVA CONGRESS, 32. 2007, Sydney. Proceedings... Sydney: Wsava Congress, 2007.

TILLEY, L. P.; SMITH, F. W. K. Consulta veterinária em 5 minutos. 2. ed. Barueri: Manole, 2008.

TORRES, P. Cardioplastía Esófago-Diafragmática como tratamiento del Megaesófago total congénito idiopático en el perro. Archivos de Medicina Veterinária, v. 32, n. 1, p. 46-52, 2000.

VALEZI, A. C. et al. Tratamento do Megaesôfago chagásico grau II por laparoscopia: experiência em 12 casos. Revista do Colégio Brasileiro de Cirurgia, v. 31, n. 3, p. 148-153, 2004.

WASHABAU, R. J.; HOLT, D. E. Pathophysiology of gastrointestinal disease. In: SLATTER, D. Textbook of small animal surgery. 3rd ed. Philadelphia: Saunders, 2003. v. 1, p. 530-552.

WASHABAU, R. J. Doenças do esôfago. In: ETTTINGER, S. J.; FELDMAN, E. C. Tratado de medicina interna veterinária: doenças do cão e gato. 5. ed. Rio de Janeiro: Guanabara Koogan, 2004. p. 1205-1214.

WILLARD, M. D. Distúrbios da cavidade oral, faringe e esôfago. In: COUTO, C. G.; NELSON, R. W. Medicina interna de pequenos animais. 3. ed. Rio de Janeiro: Elsevier, 2006. p. 398-399.

Recebido: 10/11/2009

Received: $11 / 10 / 2009$

Aprovado: 03/05/2010

Approved: 05/03/2010 